\title{
THEODORO AUGUSTO RAMOS - A BRAZILIAN CONTRIBUTION TO THE MODEL OF THE ATOM*
}

\author{
Lavinel G. Ionescu $a, b$ and Luis Alcides Brandini De Boni $b$ \\ SCIENCO Scientific Consulting Services ${ }^{a}$ \\ Huntington Beach, California, USA \\ $\&$ \\ Departamento de Química Pura, Faculdade de Química $b$ \\ Pontificia Universidade Católica do Rio Grande do Sul \\ Porto Alegre, RS, BRASIL
}

\begin{abstract}
The present article discusses the contribution of Theodoro Augusto Ramos, A Brazilian mathematician, to the model of the atom. In a paper entitled "The Theory of Relativity and the Spectral Lines of Hydrogen", presented to the Brazilian Academy of Sciences in November 1923, Theodoro Ramos analyzed the fine spectra of the hydrogen atom using the principles of the general theory of relativity. His results represent an improvement of the Bohr-Sommerfeld model.

\section{RESUMO}

O presente artigo trata da contribuição de Theodoro Augusto Ramos, um matemático brasileiro, para o modelo atômico. Num trabalho com titulo "A Theoria de Relatividade e as Raias Espectraes do Hydrogênio", apresentado perante a Academia Brasileira de Ciências em Novembro de 1923, Theodoro Ramos analizou o espectro fino do átomo de hidrogênio usando a teoria de relatividade geral.Os seus resultados representam uma melhoria do Modelo de Bohr-Sommerfeld.
\end{abstract}

KEYWORDS. Hydrogen Atom, Atomic Models, Fine Spectra, Theory of Relativity.

Theodoro Augusto Ramos (1895-1935) was one of the most notable and productive Brazilian mathematicians of his time. He graduated in civil engineering in 1917 from the Escola Politécnica of Rio de Janeiro, one of the traditional engineering schools of Latin America, founded as a military institution in 1792, soon after the arrival of the Portuguese Court in Brazil.

- Paper presented in part at the XLIV Brazilian Congress of Chemistry (Associação Brasileira de Química - ABQ), Fortaleza, Ceará, Brazil, September 20-24, 2004. 
SOUTH. BRAZ. J. CHEM., Vol.13, No. 13, 2005.

Brazilian Contribution to the lodel of the Atom

He obtained the Doctoral Degree in Physical and Mathematical Sciences from the same institution and his thesis was entitled "On Functions of Real Variables". During the same year he accepted a position in mathematics in the Engineering School (Escola Politécnica) of São Paulo. It was in São Paulo that Theodoro Ramos made his more important contributions to science and mathematics. He published various articles dealing with mathematical physics and several textbooks on advanced mathematics.

He played an important role in the establishment of what is now the University of São Paulo and was a member of the Organizing Committee named by the Governor Armando Oliveira Salles. Theodoro Ramos was the first Dean of the Faculty of Philosophy, Sciences and Letters and was instrumental in inviting and bringing to Brazil during the early 1930's a large number of European scientists in mathematics, physics, chemistry and biology.

According to well-informed sources, Theodoro Ramos spent some time working with Niels Bohr in Copenhagen and was familiar with scientific circles in Europe.

The present paper deals with the contribution of Theodoro Augusto Ramos to the model of the atom. In November 1923, he presented a work entitled "The
Theodoro Augusto Ramos (1895-1935)

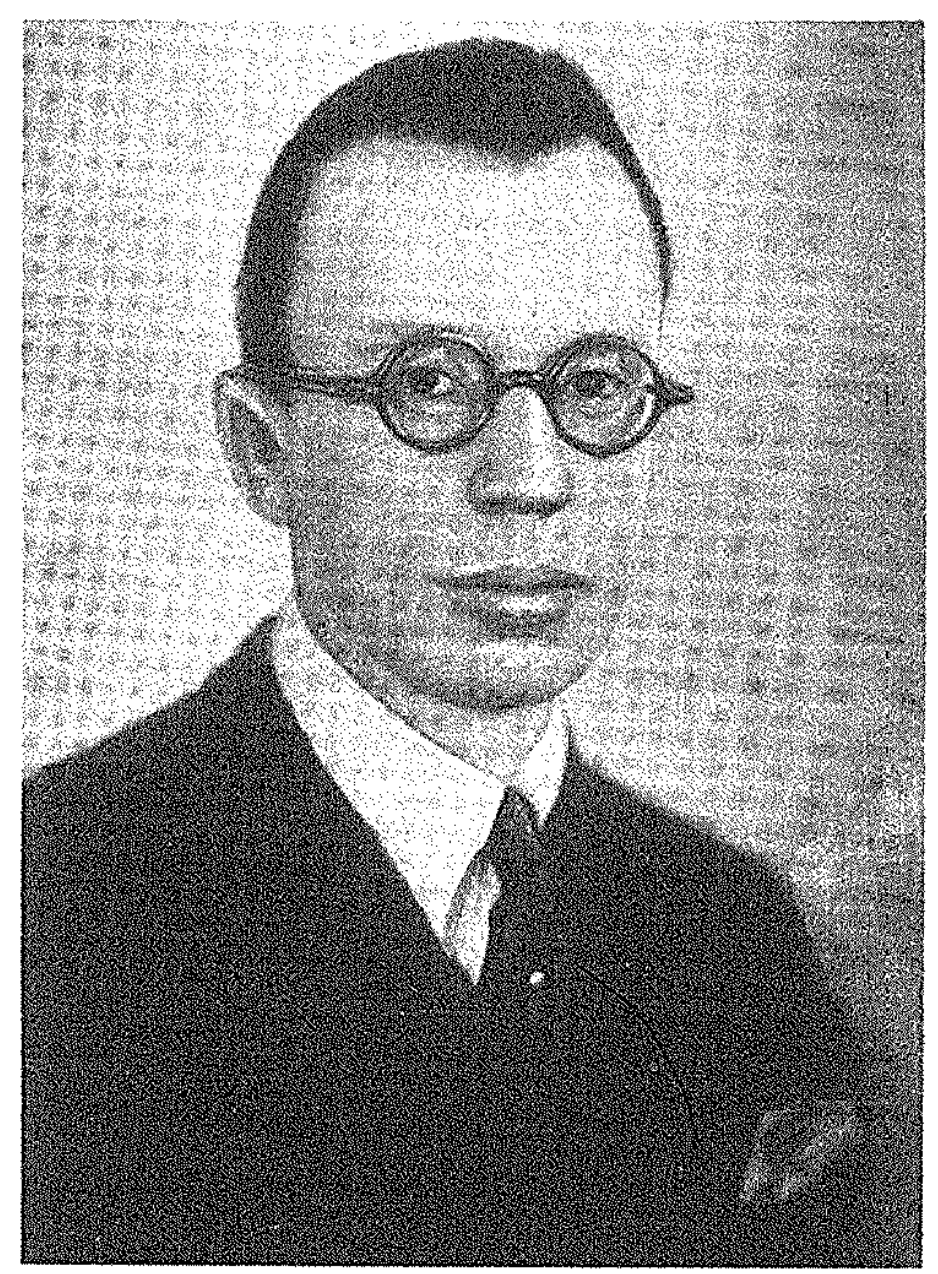
Spectral Lines of Hydrogen" to the Brazilian Academy of

Sciences. The article was published six years later in the first issue of the Annaes da Academia Brasileira de Sciencias in 1929. Theodoro Ramos analyzed the fine spectra of the hydrogen atom using the principles of the general theory of relativity. Earlier, in 1923, Arnold Sommerfeld had explained the splitting of the Balmer lines of hydrogen analyzing the movement of the electron around the nucleus using the restricted theory of relativity. 
SOUTH. BRAZ. J. CHEM., Vol.13, No. 13, 2005.

L.G. Ionescu \& L.A. B. De Boni

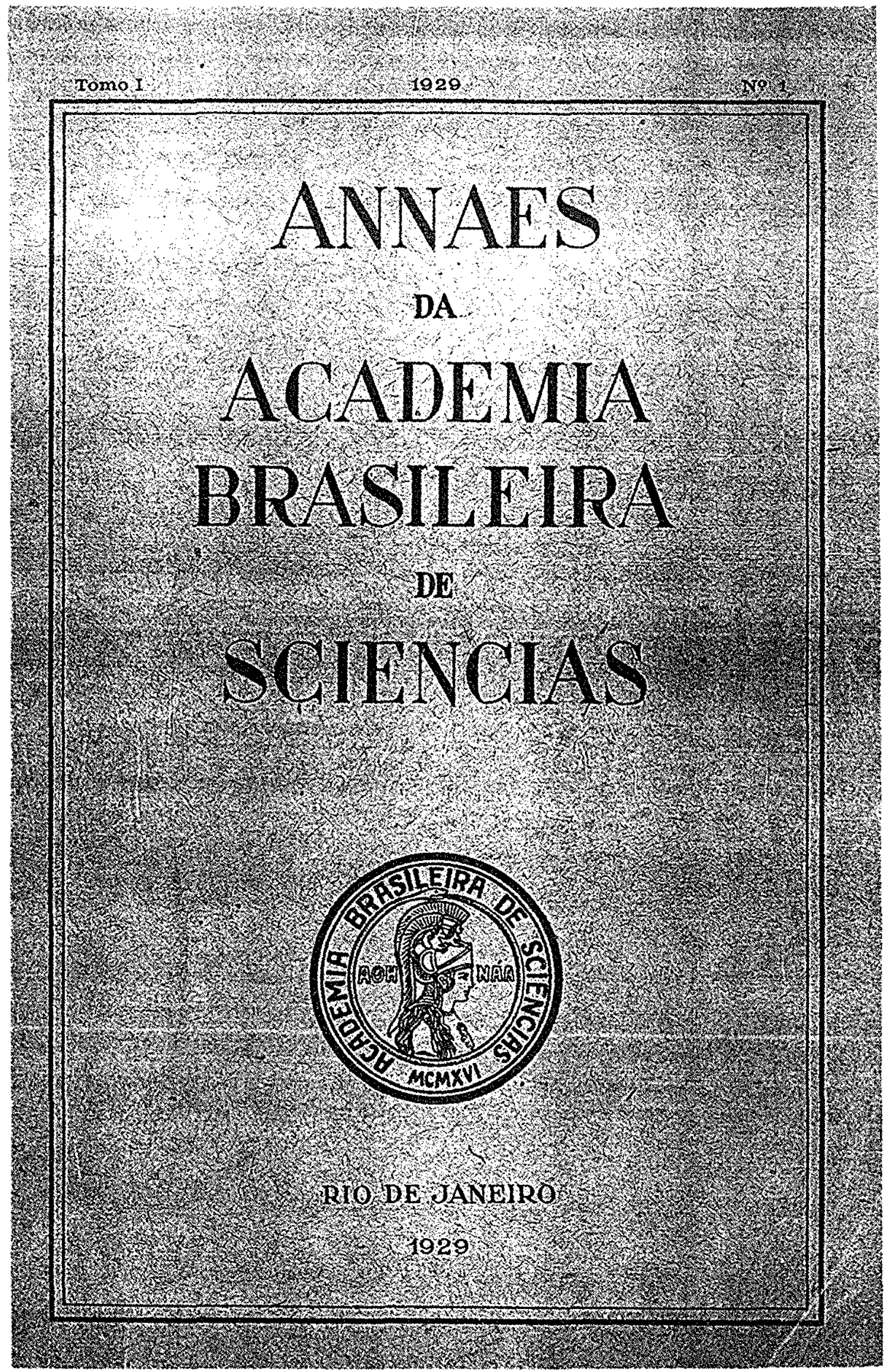


SOUTH. BRAZ. J. CHEM., Vol.13, No. 13, 2005.

Brazilian Contribution to the rodel of the Atom

\section{A THEORIA DA RELATIVIDADE E AS RAIAS ESPECTRAES DO HYDROGENIO}

THEODORO RAMOS

Sommerfeld conseguiu explicar o desdobramento das raias da série classica de Balmer relativa ao hydrogenio (1), estudando o movimento dos electrons en torno do nucleo positivo sob o ponto de vista da theoria da relatividade restricta (2). Sommerfeld suppoz um campo de Minkowski e desprezou o movimento do nucleo. G. Darwin (3), estudou a influencia deste deslocamento e achou um termo correctivo desprezivel para o afastamento das raias do "doublet".

Neste pequeno trabalho vamos abordar o problema sob o ponto de vista da theoria da relatividade generalizada.

O nucleo positivo será assimilado a uma esphera de raio $\alpha$ e de massa $M$ e cuja carga electrostatica é E. Teremos um campo com symetria espherica em que são nullas as componentes do potencial vector.

O espaço-tempo no exterior da esphera será definido por (4)

(1) Sommerfeld "La constitution de l'atome et les raies spectrales" t. 2, 1923.

(2) Mostramos em um trabalho anterior que o mesmo resultado póde ser obtido modificando ligeiramente o potencial electrostatico.

(3) C. G. Darwin, Phil. Mag. (1920), de accordo com a citação de Sommerfeld, op. cit., pg. 568, t. 2.

(4) Deve-se este ds'2 a H. Vanderlinden. Consulte-se a pg. 95 do excellente tratado de Th. De Donder "La Gravifique Einsteinienne". Este autor emprega um outro systema de constantes.

Ann. da Acad. Brasileira de Sciencias 
SOUTH. BRAZ. J. CHEM., Vol.13, No. 13, 2005.

Brazitian Contribution to the Model of the Atom

\section{A THEORIA DA RELATIVIDADE E AS RAIAS ESPECTRAES DO HYDROGENIO}

THEODORO RAMOS

Sommerfeld conseguiu explicar o desdobramento das raias da série classica de Balmer relativa ao hydrogenio (1), estudando o movimento dos electrons en torno do nucleo positivo sob o ponto de vista da theoria da relatividade restricta (2). Sommerfeld suppoz um campo de Minkowski e desprezou o movimento do nucleo. G. Darwin (3), estudou a influencia deste deslocamento e achou um termo correctivo desprezivel para o afastamento das raias do "doublet".

Neste pequeno trabalho vamos abordar o problema sob o ponto de vista da theoria da relatividade generalizada.

O nucleo positivo será assimilado a uma esphera de raio $\alpha$ e de massa $M$ e cuja carga electrostatica é $E$. Teremos um campo com symetria espherica en que são nullas as componentes do potencial vector.

O espaço-tempo no exterior da esphera será definido por (4)

(1) Sommerfeld "La constitution de l'atome et les raies spectrales" t. $2,1923$.

(2) Mostramos em um trabalho anterior que o mesmo resultado póde ser obtido modificando ligeiramente o potencial electrostatico.

(3) C. G. Darwin, Phil. Mag. (1920), de accordo com a citação de Sommerfeld, op. cit., pg. 568 , t. 2 .

(4) Deve-se este ds"2 a H. Vanderlinden. Consulte-se a pg. 95 do excellente tratado de Th. De Donder "La Gravifique Einsteinienne". Este autor emprega um outro systema de constantes.

Ann. da Acad. Brasileira de Sciencias 


$$
\begin{gathered}
d s^{2}=-\frac{d r^{2}}{1-\frac{\gamma}{r}+\frac{\varepsilon^{2}}{r^{2}}}-r^{2}\left[d \theta^{2}+\operatorname{sen}^{2} \theta d \varphi^{2}\right]+ \\
+c^{2}\left(1-\frac{\gamma}{r}+\frac{\varepsilon^{2}}{r^{2}}\right) d t^{2}
\end{gathered}
$$

em que $\quad \gamma=\frac{2 f M}{c^{2}}+\frac{\varepsilon^{2}}{a} \quad$ e $\varepsilon^{2}=\frac{f E^{2}}{c^{4}}$

$f$ designando a constante de Gauss e $c$ a velocidade da luz.

O movimento do electron de massa $m$ e de carga $e$ póde ser estudado no espaço e no tempo com o auxilio do principio generalizado da conservação da quantidade de movimento e da energia.

Ponhamos $\quad L=m c^{2}[1-\gamma]+\frac{e E}{r}$

em que $\quad V=\frac{1}{c} \frac{d s}{d t}$

e consideremos o tensor $\quad \omega_{\delta}=p_{x} \delta v+p_{\varphi} \delta_{\varphi}+p_{\theta} \delta \theta-W \delta t$, cujas componentes de espaço são as quantidades de movimento generalizadas (1)

$$
p_{\mathrm{r}}=\frac{\delta L}{\delta r^{\prime}}, \quad p_{q}=\frac{\delta L}{\delta \varphi^{\prime}}, p_{\theta}=\frac{\delta L}{\delta \theta^{\prime}}
$$

(os accentos designando as derivadas em relação a $t$ ), e cuja componente de tempo é a energia total generalizada

$$
W=r^{\prime} \frac{\delta L}{\delta r^{\prime}}+\varphi^{\prime} \frac{\delta L}{\delta \varphi^{\prime}}+\theta^{\prime} \frac{\delta L}{\delta \theta^{\prime}}-L
$$

As equações differenciaes do movimento serão obtidas exprimindo que ellas admittem como invariante integral $\int_{\mathrm{c}} \omega_{\delta}$ extendida a um contorno fechado qualquer no espaço a 7 dimensões $\left(r, \varphi, \theta, p_{\mathrm{r}}, p_{\phi}, p_{\theta}, t\right)$. mentos".

(1) Alguns autores dão a estas expressões a denominação de "mo- 
Obtem-se assim, mediante um calculo classico, as condiçôes

$$
\begin{gathered}
a p_{\mathrm{r}}+\frac{\delta W}{\delta r} d t=0 \\
\vdots d r+\frac{\delta W}{\delta p_{\mathrm{r}}} d t=0
\end{gathered}
$$

e mais 4 equações analogas para $\varphi$ e $\theta$, e tàmbem

$$
-d W+\frac{\delta W}{\delta t} d t=0
$$

Como $W$ não depende explicitamente de $\varphi$ e de t, tira-se immediatamente $\frac{d p_{\varphi}}{d t}=0, d W \equiv 0$

$$
p_{q}=\text { const. }=p \quad \text { e } \quad W=\text { const. } ;
$$

vem tambem $\frac{d_{p_{0}}}{d s}=\frac{\delta W}{\delta \theta} \frac{d t}{d s}$

que desenvolvida dá

$$
\frac{d}{d s}\left(r^{2} \frac{d \theta}{d s}\right)=r^{2} \operatorname{sen} \theta \cos \theta\left(\frac{\ddot{d} \phi}{d s}\right) \ddot{~}
$$

Estas equações permittem concluir que a energia total generalizada é constante e que a trajectoria póde ser considerada como pertencente ao plano $\theta=\frac{\pi}{2}$ :

$$
\text { As equações } \quad p_{\varphi}=p \quad p^{\prime} \quad W=\text { const. }
$$

dão, então, $\quad m e$ $2 \frac{d \varphi}{d s}=p$

$$
c\left[1-\frac{\gamma}{r}+\frac{\varepsilon^{2}}{r^{2}}\right] \frac{d t}{d s}=\frac{W}{m c^{2}}+\frac{e E^{2}}{m c^{2} r^{2}}+1
$$

Tem-se, tambem,

$$
p_{\mathrm{r}}=m e \frac{d r}{d s}\left[1-\frac{\gamma}{r}+\frac{\varepsilon^{2}}{r^{2}}\right]^{-1}
$$

Ann. da Acad. Brasileira de Sciencias 


$$
\begin{aligned}
c^{2}\left[1-\frac{\gamma}{r}+\frac{\varepsilon^{2}}{r^{2}}\right] & \left(\frac{\dot{d} t}{d s}\right)^{2}-\left[1-\frac{\gamma}{r}+\frac{\varepsilon^{2}}{r^{2}}\right]^{-1}\left(\frac{d r}{d \dot{s}}\right)^{2}- \\
& -r^{2}\left(\frac{d \varphi}{d s}\right)^{2}=1 .
\end{aligned}
$$

Eliminando $\frac{d t}{d s}, \frac{d r}{d s} e \frac{d \varphi}{d s}$ entre estads 4 equações, vem

$$
\begin{aligned}
& p_{\mathrm{r}}^{2}=\left[\frac{w}{c}+\frac{e E}{r}+m c\right]^{2}\left[1-\frac{\gamma}{r}+\frac{\varepsilon^{2}}{r^{2}}\right]^{-2} \\
& -m c^{2}\left[1-\frac{\gamma}{r}+\frac{\varepsilon^{2}}{r^{2}}\right]^{-1}-\frac{p^{2}}{r^{2}}\left[1-\frac{\gamma}{r}+\frac{\varepsilon^{2}}{r^{2}}\right]^{-1}
\end{aligned}
$$

Desenvolvendo em série

$$
\left[1-\frac{\gamma}{r}+\frac{\varepsilon^{2}}{r^{2}}\right]^{-1} \text { e }\left[1-\frac{\gamma}{r}+\frac{\varepsilon^{2}}{r^{2}}\right]^{-2} \text { conservando }
$$

sómente os primeiros termos do desenvolvimento (o que é permittido pois $\frac{\gamma}{r} \cdot e \frac{\varepsilon^{2}}{r^{2}}$ são muito pequenos) vem parà $p_{x}^{2}$ a expressão approximada

$$
\begin{gathered}
p_{\mathrm{r}}^{2}=\left(2 m W+\frac{W^{2}}{c^{2}}\right)+2\left[m e E+\frac{e E W}{e^{2}}-\frac{m c^{2} \gamma}{2}\right] \frac{1}{r}+ \\
+\left[\frac{e^{2} E^{2}}{c^{2}}-p^{2}\right] \frac{1}{r^{2}}
\end{gathered}
$$

As condições de estabilidade da trajectơria são

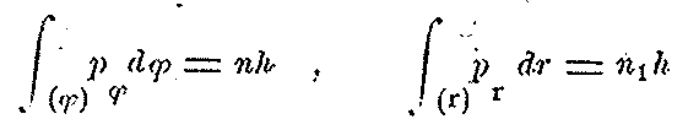

em que $n$ e $n_{1}$ designam 2 numeros inteiros e $h$ é a constante universal de Planck. As integrações devem ser extendidas á todo o dominio de variação de $\varphi_{r}$ e de respectivamente. 
SOUTH. BRAZ. J. CHEM., Vol.13, No. 13, 2005.

Brazilian Contribution to the Model of the Atom

A primeira çondição dá $p=\frac{h \hbar h}{2 \ddot{x}}$.

Quanto á segunda, tem-se

$$
r=\int_{(\mathrm{r})} l \overline{A+2 \frac{B}{r}+\frac{C}{r^{2}}} d r=n_{1} h
$$

em que

$A=2 m W+\frac{W^{2}}{O^{2}}, B=m e E+\frac{e E W}{e^{2}}-\frac{m c^{2} \gamma}{2}, C=-\frac{n \cdot h^{2}}{4 \pi}+\frac{e^{2} E^{2}}{c^{2}}$

As integraes do typo de $\int \mathrm{já}$ foram calculadas por Sommerfeld.

Encontra-se $\quad J=-2 \pi i\left(c-\frac{B}{\sqrt{A}}\right)=n_{1} \mu$

$$
\begin{gathered}
-2 \pi i \theta=-n \sqrt{n^{2}-u^{2}}, \quad \alpha=\frac{2 \pi e^{2}}{h c} \\
\frac{B}{V A}=\frac{e E\left(1+\frac{W}{m c^{2}}\right)-\frac{c^{2} \gamma}{2}}{c\left[\left(1+\frac{W}{m c^{2}}\right)^{2}-1\right]^{1 / 2}}, \quad u=a \frac{B}{e}
\end{gathered}
$$

Obtem-se a relação approximada

$$
\begin{gathered}
{\left[4 \pi^{2} e^{2} E^{2}+c^{2} n^{2}\left(n_{i}+\sqrt{n^{2}-u^{2}}\right)^{2}\right]\left(1+\frac{W}{m c^{2}}\right)^{2}-} \\
-4 \pi^{2} c^{2} \gamma e E\left(1+\frac{W}{m c^{2}}\right)-c^{2} h^{2}\left(n_{1}+V \overline{n^{2}-u^{2}}\right)^{2}=0
\end{gathered}
$$

Com grande approximação póde-se escrever

$$
\begin{aligned}
& 1+\frac{W}{m c^{2}}=\left[1+\frac{u^{2}}{\left(n_{1}+\sqrt{\left.n-n^{2}\right)^{2}}\right.}\right]^{-1 / 2} \times \\
& \times\left[1+\frac{\frac{\pi^{2} \gamma^{2} e^{2}}{2 h^{2}} u^{2}\left(n_{1}+\sqrt{n^{2}-u^{2}}\right)^{-4}}{u^{2}\left(n_{1}+\sqrt{n^{2}-u^{2}}\right)^{-2}+1}\right]+\frac{\frac{\pi \gamma^{2}}{h} u\left(n_{1}+\sqrt{n^{2}-u^{2}}\right)^{-2}}{u^{2}\left(n_{1} \sqrt{n^{2}-u^{2}}\right)^{-2}+1}
\end{aligned}
$$

Ann. da Acat. Brasileira de Sciencias 
Desenvolvendo em série os radicaes, vem

$$
\begin{aligned}
& {\left[1+\frac{u^{2}}{\left(n_{1}+\sqrt{\left.n^{2}-u^{2}\right)^{2}}\right.}\right]^{-1}=1-\frac{1}{2} \frac{u^{2}}{\left(n+n_{1}\right)^{2}}-} \\
& -\frac{1}{2} \frac{u^{4}}{\left(n+n_{1}\right)^{4}}\left(\frac{1}{1}+\frac{n_{1}}{n}\right)+\cdots \cdots \\
& \frac{\frac{\pi^{2} \gamma^{2} c^{2}}{2 h^{2}} u^{2}\left(n_{1}+\sqrt{n^{2}-u^{2}}\right)-4}{u^{2}\left(n_{1}+\sqrt{\left.n^{2}-u^{2}\right)}=-1\right.}= \\
& =\frac{\pi^{2} \gamma^{2} c^{2}}{2 h^{2}}\left[\frac{u^{2}}{\left(n+n_{1}\right)^{4}}+\frac{2 n_{1}+n}{n\left(n+n_{1}\right)^{6}} n^{4}+\ldots . .\right] \\
& \frac{\frac{\pi e^{e}}{h} u\left(n_{1}+\sqrt{n_{2}-u_{2}}\right)^{-2}}{u^{2}\left(n_{1}+\sqrt{n_{2}-u_{2}}\right)^{-2}+1}= \\
& =\frac{\pi \gamma c}{h}\left[\frac{u}{\left(n+n_{1}\right)^{2}}+\frac{u^{3}}{\left(n+n_{1}\right)^{4}} \cdots n_{1}+\cdots\right]
\end{aligned}
$$

A frequencia $\nu$ da radiação emittida (ou absorvida) quando $o$ electron passa de uma orbita á qual corresponde a energia $W_{k}$ para outra á qual se refere a energia $W_{n}$ é dada pela equação de Planck-Einstein

$$
h v=W_{k}-W_{n}
$$

Vem

$$
\nu=\left[n, n_{1}\right]-\left[k, k_{1}\right]
$$


SOUTH. BRAZ. J. CHEM., Vol.13, No. 13, 2005.

Brazilian Contribution to the Model of the Atom

em que

$$
\begin{gathered}
{\left[n, n_{1}\right]=R \frac{E}{e}\left\{\left(\frac{E}{e}-\frac{2 \pi \gamma c}{h \alpha}\right) \frac{1}{\left(n+n_{1}\right)^{2}}-\right.} \\
\left.-\frac{2 \pi \gamma c}{h} \frac{E}{e} \frac{\alpha}{\left(n+n_{1}\right)^{4}} \frac{n_{1}}{n}+\frac{\alpha^{2}}{\left(n+n_{1}\right)^{4}}\left(\frac{E}{e}\right)^{3}\left(\frac{1}{4}+\frac{n_{1}}{n}\right)+\cdots\right\} \\
R=\frac{2 \pi^{2} m e^{4}}{h^{3}}
\end{gathered}
$$

Về-se que o termo achado por Sommerfeld

$$
R\left(\frac{E}{e}\right)^{2} \frac{1}{\left(n+n_{1}\right)^{2}}+\frac{1^{\prime}}{4} \frac{\alpha^{2} R}{\left(n+n_{1}\right)^{4}}\left(\frac{E}{e}\right)^{4}
$$

soffre uma diminuição cuja parte principal é dada pela quantidade

$$
-\frac{2 \pi \gamma c}{h \alpha} R \frac{E}{e} \frac{1}{\left(n+n_{1}\right)^{2}}
$$

Quanto ao termo que rege a estructura do "doublet", soffre tambem uma diminuição correspondente a

$$
-\frac{2 \pi \gamma^{c}}{h} \propto R\left(\frac{E}{j^{e}}\right)^{3} \frac{n_{1}}{n} \frac{1}{\left(n+n_{1}\right)^{4}} .
$$

A correcção do termo principal representa, para o hydrogenio, uma fracção do termo de Sommerfeld $\frac{1}{4} \frac{\alpha^{2} R}{\left(n_{1}+n\right)^{4}}$ da ordem de grandeza de $\frac{\gamma c}{h a^{3}}$ ou 10-7 (1), e não póde, portanto, ser, actualmente, submettida ao "contrôle" experimental.

(1) Adoptando para as constantes os valores que se acham na obra de Sommerfeld, e admittindo com Rutherford que é de 10-16 a ordem de grandeza do raio do nucleo positivo.

Ann. da Acad. Brasileira de Sciencias 
SOUTH. BRAZ. J. CHEM., Vol.13, No. 13, 2005.

L.C. Ionescu \& L.A.B. De Boni

Quanto á correç̧ão relativa á estructura do "doublet", ella é uma fracção da ordem de $10-1 \%$ do termo correspondente achado por Sommerfeld; trata-se, pois, de uma modificação desprezivel. $E$ ', entretanto, interessante constatar que Paschen em suas experiencias achou para o afastamento do "doublet" do hydrogenio um valor ligeiramente inferior ao que foi calculado por Sommerfeld. A experiencia confirma, pois, uma correcçã̀o tendo o mesmo sentido da que achamos; a ordem de grandeza é, porém, differente.

Terminando esta nota assignalaremos que o estudo da orbita do electron póde ser effectuado com o auxilio das funcções ellipticas. A variavel $p$ é dada em funç̧ão de $r$ por uma integral que contém um radical do $4 .^{\circ}$ gráo em $\frac{1}{\tau}(2)$.

Sessão de Novembro de 1923.

(2) En um campo de Schwarzschild encontra-se um radical do 3. grảo.

T. $1, n .^{\circ} 1$, Marso de 1929 
SOUTH. BRAZ. J. CHEM., Vol.13, No. 13, 2005.

Brazilian Contribution to the Model of the Atom

Theodoro Ramos approached the problem from the point of view of the general theory of relativity. It is a rather complex mathematical treatment that analyzes the movement of the electron around the nucleus and it involves differential equations with seven coordinates $\left(r, \theta, \varphi, \rho_{r}, \rho_{\theta}, \rho_{\varphi}, t\right)$. Since the development is essentially mathematical, we reproduced the original article in entirety in Portuguese.

As can be seen from the analysis of the treatment, the results obtained by Theodoro Ramos explained a little better the splitting of the doublet and represents an improvement of the Bohr-Sommerfeld model of the atom.

ACKNOWLEDGEMENT. Finnancial support received by LGI from Sarmisegetusa Research Group (SRG), Santa Fe, New Mexico, USA, is gratefully acknowledged.

\section{REFERENCES}

1. T. A., Ramos, Ann. Acad. Bras.Ci., I(1), 20-27(1929).

2.A.Clássico, Spectrum-J.Bras.Ci., 1(2), 46-48 (1981).

.3.. M. Guimarães Ferri and S. Motoyama. "Hstória das Ciências no Brasil". EDUSP, São Paulo, SP, Brasil, Vol.2, pp. 44-45, 1979.

4. L.G. Ionescu and L.A. B. De Boni, Tchê Quimica, 2(1), 30-37 (2005).

5. F. De Azevedo, "As Ciência no Brasil", Editora Melhoramentos, São Paulo, Brasil, 1955.

OPEN ACCESS. This article is licensed under a Creative Commons Attribution 4.0 (CC BY 4.0) International License, which permits use, sharing, adaptation , distribution , and 